\title{
Malignant vasovagal syncope: a randomised trial of metoprolol and clonidine
}

\author{
Mauro Biffi, Giuseppe Boriani, Paolo Sabbatani, Gabriele Bronzetti, Lorenzo Frabetti, \\ Romano Zannoli, Angelo Branzi, Bruno Magnani
}

\begin{abstract}
Objective-To evaluate the efficacy of head up tilt guided treatment with metoprolol and clonidine in preventing the recurrence of syncope in patients with malignant vasovagal syncope.

Patients-20 patients (9 men and 11 women, mean age 33 (SD 17), range 14 to 62 years) with severe symptoms.

Design-Randomised double blind crossover trial; efficacy was assessed by head up tilt testing.

Results-Metoprolol was more effective than clonidine in abolishing syncope $(19 / 20 v 1 / 20, P<0.001)$ but clonidine showed some beneficial effects on time to syncope and severity of hypotension in $\mathbf{1 2}$ patients. During an average follow up of 15 (3) months there was a significant reduction in the recurrence of symptoms compared with the previous year in patients who had tilt up guided treatment (18 metoprolol, 1 clonidine).

Conclusions-Treatment guided by head up tilting is a reliable method of treating patients with malignant vasovagal syndrome. Metoprolol was an effective long term treatment for preventing syncope. High doses were more effective and a careful dose titration period helped to minimise withdrawal symptoms and side effects.
\end{abstract}

(Heart 1997;77:268-272)

Keywords: neurocardiogenic syncope; head up tilt test; $\beta$ blockers; clonidine

Vasovagal syncope is a common clinical problem forming the pathophysiological basis of syncope in up to $25 \%$ of patients seen at an emergency department for this condition, ${ }^{1}$ and it may account for nearly half the cases of syncope. $^{2}$

Although it usually has a favourable prognosis, it may be a highly limiting clinical problem in a particular subset of patients among whom syncopal recurrences are frequent and without prodromic symptoms, or have a traumatic outcome. For this subgroup of patients the term "malignant vasovagal syncope" has been proposed to identify these characteristics. ${ }^{3}$ The treatment of these highly symptomatic patients is necessary to avoid dangerous injuries and to improve their quality of life.

The treatment of vasovagal syncope is controversial and different approaches have been proposed. Among these, $\beta$ blockers, cardiac pacing, disopyramide, clonidine, and scopolamine are the most frequently employed. ${ }^{4-12}$ In this study we investigated the efficacy of metoprolol and clonidine in a randomised double blind crossover study to prevent syncope recurrence in a highly symptomatic group of patients with "malignant" vasovagal syndrome.

Since there is clinical evidence for the use of metoprolol, ${ }^{4510}$ we tested the hypothesis that a sympathetic modulator such as clonidine might also be effective, as reported by Fitzpatrick et al in a randomised trial. ${ }^{8}$ The use of clonidine has strong pathophysiological support because of its partial selective $\alpha_{2}$ agonist activity ( $\alpha_{2}$ receptors predominate in large capacitance vessels, and clonidine would significantly reduce venous capacitance, thus decreasing the likelihood of triggering the vasovagal reflex following gravitational stress). Beneficial effects were observed by Robertson et al in patients with severe orthostatic hypotension, who showed a great improvement in systolic blood pressure and functional capacity with clonidine treatment. ${ }^{13}$

\section{Methods}

Patients with frequent recurrences of syncope (one or more per month) or injuries caused by syncopal events due to vasovagal syncope seen at our hospital from March 1993 to May 1995 were screened for enrolment in the study.

Exclusion criteria were a clinical history of asthma or chronic obstructive airways disease, atrioventricular block greater than first degree, sinus rate less than 40 beats $/ \mathrm{min}$, structural heart disease with contraindications for $\beta$ blocking agents, hypersensitivity to $\beta$ blockers, and patients' refusal to participate.

Twenty patients fulfilled the entry criteria for randomisation (more than 12 syncopal episodes or more than one syncopal event with traumatic outcome in the previous year, or both). The diagnosis of vasovagal syncope was ruled out by a standardised cardiological and neurological approach in all the patients. The work up consisted of clinical examination, laboratory screening, 12 lead electrocardiogram (ECG), cross sectional echocardiography, and 24-hour Holter monitoring. Electrophysiological study was performed when clinically indicated.

Vasovagal syncope was confirmed by head up tilt test (HUT) according to a previously published classification ${ }^{14}$; after baseline HUT, patients were randomised to receive either \\ Cardiology, Policlinico \\ $S$ Orsola, University of
}




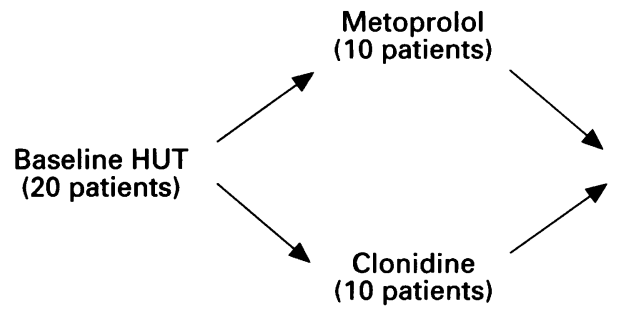

metoprolol or clonidine in a double blind crossover manner. The study consisted of two distinct phases: the acute drug evaluation phase, and the chronic treatment phase. During the acute phase, the efficacy of both treatments was assessed in a double blind, randomised, crossover manner. During the chronic phase the study was carried out in single blind manner.

Patients were instructed by the referral physician to take the drugs orally on a progressively increasing dose schedule over a 10 day period; HUT was then repeated on the 15 th day. A five-day wash out period was allowed, and then patients crossed over to the second treatment with a similar dosage titration period; on the 15 th day, HUT was repeated as in phase 1 (figure).

Both patients and physicians performing the HUT were blinded to the treatment taken by the patients. Metoprolol and clonidine were given twice daily, increasing from $50 \mathrm{mg}$ up to $400 \mathrm{mg}$ daily for metoprolol and from $0 \cdot 150$ $\mathrm{mg}$ up to $1.2 \mathrm{mg}$ daily for clonidine.

Patients were instructed to report potential side effects of the study drug; dosage titration was limited by the onset of intolerable side effects or excessive bradycardia (resting heart rate $<40$ beats $/ \mathrm{min}$ ).

\section{HUT PROTOCOL}

HUT was performed by a standardised protocol in our laboratory. Tests were performed at 10:30 in a comfortable quiet room at $22^{\circ} \mathrm{C}$; cardiac rhythm was continuously monitored on a Marquette unit (Case 12) and blood pressure by a Finapress unit (Ohmeda 2300).

The HUT protocol consisted of two phases: basal HUT and a pharmacological test. Basal HUT was performed as follows: after $10 \mathrm{~min}$ utes of rest, the bed was tilted to $70^{\circ}$ for 25 minutes; in case of negative response, the pharmacological test ensued. The pharmacological test was accomplished by isoprenaline infusion in five progressive steps, from 1 up to $5 \mu \mathrm{g} / \mathrm{min}$ $(0.15$ to $0.07 \mu \mathrm{g} / \mathrm{kg} / \mathrm{min})$ at $80^{\circ}$ tilt. Each step consisted of five minutes supine and $10 \mathrm{~min}$ utes tilt at constant infusion rate.

Our protocol was based on data in published reports. ${ }^{3-5} 111215-17$ The most recent studies confirm the sensitivity, specificity, and reproducibility of HUT results by this degree of table tilting and test duration, either in the basal condition or during isoprenaline testing. ${ }^{18} 19$
After the acute drug evaluation phase was completed, patients were allocated to the chronic treatment phase on the study drug which had changed the baseline HUT to a negative response, or on study drug 2 if they responded to both. If neither drug was effective, the patient was treated on the basis of the referring physician's judgement.

\section{Statistical analysis}

Results are given as mean (SD). The haemodynamic effect of the two study drugs was compared by analysis of variance.

The effect of the two study drugs during the acute phase (the number of patients who respectively converted from a positive to negative HUT) was evaluated by contingency tables.

The results during the follow up period were evaluated by McNemar's test.

\section{Results}

Twenty of 203 consecutive patients referred to our outpatient clinic for syncope of unexplained origin fulfilled the clinical entry criteria for enrolment into the study. Mean age was 33 (17) (range 14 to 62) years; there were nine males and 11 females. None of the patients had any relevant clinical abnormalities or organic heart disease. All 20 patients had negative tests on the preliminary diagnostic work up, but vasovagal syncope was confirmed by a positive baseline HUT in all cases. Among these 20 patients, 14 had had more than 12 syncopal attacks in the year preceding baseline HUT, while six had two traumatic syncopes. Among the latter there was a mean of $3(1 \cdot 3)$ (range 2 to 4 ) syncopes in $4(2 \cdot 8)$ months (range 1 to 9 ) since the first syncopal episode.

Baseline HUT elicited syncope in all the patients, in $11(55 \%)$ during basal HUT, and in nine $(45 \%)$ during pharmacological testing. Among these latter, syncope was reproduced at an isoprenaline infusion rate of $1.6(0.9)$ $\mu \mathrm{g} / \mathrm{min}$ (range 1 to $3 \mu \mathrm{g} / \mathrm{min}$ ).

At baseline HUT, $14 / 20$ patients (70\%) showed a vasodepressive pattern of syncope, $4 / 20(20 \%)$ a mixed pattern, and $2 / 20(10 \%)$ a cardioinhibitory pattern (table 1 ).

During the acute phase, HUT was considered negative if both basal HUT and the five 
Table 1 Head up tilt responses at baseline and during treatment

\begin{tabular}{llllc}
\hline & Vasodepressive & Mixed & Cardioinhibitory & Negative \\
\hline Baseline & 14 & 4 & 2 & 0 \\
Metoprolol & 0 & 1 & 0 & $19^{\star}$ \\
Clonidine & 11 & 7 & 1 & 1 \\
\hline
\end{tabular}

$\star \mathrm{P}<0.001 v$ baseline and clonidine.

Table 2 Haemodynamic variables at baseline and during drug evaluation. Values are means (SD) and range

\begin{tabular}{|c|c|c|c|}
\hline & Baseline & Metoprolol & Clonidine \\
\hline \multirow{2}{*}{$\begin{array}{l}\text { Heart rate (beats/min) } \\
\text { (range) } \\
\text { Mean blood pressure } \\
\text { (mm Hg) } \\
\text { (range) }\end{array}$} & $\begin{array}{l}78(12) \\
(60 \text { to } 105)\end{array}$ & $\begin{array}{l}58(8)^{\star} \\
(44 \text { to } 73)\end{array}$ & $\begin{array}{l}60(8)^{\star} \\
(46 \text { to } 74)\end{array}$ \\
\hline & $\begin{array}{l}105(10) \\
(87 \text { to } 126)\end{array}$ & $\begin{array}{l}100(14) \\
(80 \text { to } 128)\end{array}$ & $\begin{array}{l}103(11) \\
(85 \text { to } 123)\end{array}$ \\
\hline
\end{tabular}

${ }^{\star} \mathrm{P}<0.005 v$ baseline (ANOVA).
Table 3 Data at follow up (15 (SD 3) months)

\begin{tabular}{lll}
\hline & Previous year & Follow up \\
\hline Syncopes & 193 & $0^{\star}$ \\
Near syncopes & 182 & $7^{\star}$ \\
\hline$\star \mathrm{P}<0.001$. &
\end{tabular}

sion (average blood pressure 48 (4) $\mathrm{mm} \mathrm{Hg}$, range 38 to 55), but no syncope; among these, six had a prolongation and two no change in time to positive HUT compared to baseline HUT. Overall, at least a partial effect was observed in $12 / 20$ patients on clonidine (60\%).

During metoprolol treatment $19 / 20$ patients converted to negative HUT; only $1 / 20$ had a persistent mixed response with no change in time to syncope compared to baseline or clonidine. This patient was taking $200 \mathrm{mg}$ of metoprolol as the maximum tolerated dose.

At repeated HUT on clonidine, a slightly higher prevalence of mixed patterns $(7 / 20)$ was observed compared to baseline HUT; this was associated with a decrease $(11 / 20)$ of vasodepressor responses (table 1).

\section{FOLLOW UP DATA}

During follow up, patients were instructed to record every syncopal and near-syncopal episode (defined as lightheadedness which required the patient to lie supine to abort syncope, or forthcoming syncope aborted by health professionals or trained personnel) and to contact the referral physician as soon as possible in case of symptom recurrence. Nineteen patients were followed up to 15 (3) months (range 11 to 24), 18 on metoprolol and one on clonidine. No syncopal episodes have been reported; near-syncopal episodes have occurred in four patients, although to a dramatically lesser extent than in the year preceding baseline HUT (table 3 ). No withdrawals occurred during the follow up period because of treatment related side effects. patient (a responder to both treatments) had negative response $(P<0.001 v$ metoprolol; table 1).

Among the 19 patients not responding to clonidine, 10 showed a favourable effect of the drug in terms of time to onset of symptoms and severity of symptoms. A prolongation of time to syncope was observed in 10 patients (six who fainted in basal conditions had a positive HUT during isoprenaline testing; four who fainted during isoprenaline testing had a positive HUT at the following isoprenaline step); no change occurred in three patients, and a shortening in time to syncope occurred in six patients. Among these six patients, two who had syncope after 25 minutes and 19 minutes at baseline HUT developed syncope after 11 minutes and 12 minutes on clonidine, respectively, whereas four who had syncope during isoprenaline testing at baseline HUT developed syncope at an earlier step of isoprenaline testing on clonidine.

No difference in baseline HUT was observed in patients who had a prolongation of time to syncope, no change, or a shortening in time to syncope with clonidine.

In terms of severity of symptoms, eight patients had severely symptomatic hypoten-

\section{Discussion}

The treatment of "malignant vasovagal syndrome" is still controversial, mainly because of a lack of controlled trials. Controlled studies may be difficult to carry out in patients with o only sporadic syncopal events; on the other hand, placebo treatment is unacceptable in severely symptomatic patients, such as our study population, especially when effective treatment may be identified by provocative tests. Tilt up guided treatment may be very helpful in assessing the effectiveness of treatments to prevent syncope recurrence in high risk subgroups. 121720

In our study, we evaluated the efficacy of metoprolol and clonidine in a randomised, double blind, crossover manner.

\section{ACUTE PHASE}

The results confirm that metoprolol is very effective in preventing syncope in these highly symptomatic patients, whereas clonidine seems less effective. However, we would emphasise that clonidine should not be con- 
sidered to be ineffective; in fact, 10 patients had a prolongation of time to syncope at HUT on clonidine, and in eight patients severe symptomatic hypotension occurred but was not followed by syncope. These results are in agreement with the findings of Fitzpatrick et $a l,{ }^{8}$ who showed that time to syncope was prolonged in a randomised trial of clonidine, atenolol, scopolamine, and placebo. In this study, clonidine and scopolamine were more effective than atenolol, which shortened time to syncope. Some important differences, however, must be noted. The number of persistent positive HUT tests was $6 / 13$ for clonidine and scopolamine, $8 / 13$ for atenolol, and $9 / 13$ for placebo. This is a much poorer response than in our study population, which appears to be a "sicker" population from the point of view of clinical presentation (in Fitzpatrick's study the average rate of syncopes was 3 (SD 4) per annum).

As pointed out by Fitzpatrick et $a l,{ }^{8}$ the lipophilic characteristics of metoprolol may be of paramount importance compared to atenolol when considering central $\beta$ blocking effects. In fact, there is growing evidence that $\beta$ blockers have central serotonin blocking properties. ${ }^{21}{ }^{22}$ Recent research has shown that the pivotal event of neuromediated hypotension-sympathetic withdrawal-may be induced by both intracerebral and systemic administration of serotonin..$^{23}{ }^{24}$ Following this concept, Grubb and Kosinski have treated patients with refractory neurocardiogenic syncope with serotonin reuptake inhibitors-fluoxetine and sertraline hydrochloride-with beneficial long term clinical effects. ${ }^{25}$ These findings provide new insight in the pharmacodynamics of $\beta$ blockers and may explain the observed differences between metoprolol and atenolol. Moreover, one important point must be kept in mind: whereas the clonidine dose used by Fitzpatrick et $a l^{8}$ was similar to that in our study $(0.742(0.67) \mathrm{mg})$, our dose of metoprolol was clearly higher than that of atenolol $(50 \mathrm{mg})$ used in that study. Apart from any special pharmacokinetic-pharmacodynamic features of atenolol, its apparent inefficacy may reflect the lower dose given. The dose of metoprolol used in our study was also higher than that reported by Sra et $a l^{45}$; in fact the success in abolishing syncope reproducibility assessed by HUT was higher in our study (95\%).

In a recent paper by Sra et $a l,{ }^{4}$ however, only patients with cardioinhibitory response were considered, and this may represent a subgroup in which prevention of vasovagal episodes may be more difficult. Moreover, the efficacy of $\beta$ blocking agents was tested by repeated HUT during steady state esmolol infusion. Although Sra et al have shown that acute intravenous esmolol administration is highly predictive of success with chronic metoprolol treatment, ${ }^{5}$ this may have caused a small underestimate of the overall efficacy of $\beta$ blockers, as observed in our patients.

Indeed, in a previous study with HUT repeated during steady state oral metoprolol administration, Sra et al themselves have reported an overall efficacy comparable to that in our study: a negative HUT test on metoprolol in $25 / 26$ patients. ${ }^{10}$ From this standpoint, it is conceivable that the prevention of the vasovagal reflex may be accomplished only with complete blockade of its afferent limb, which may require full adrenergic blockade over several days of consistent drug administration. In the same way, the relative weight of central antiadrenergic and serotonin blocking effects is much more highly expressed at high metoprolol doses.

In a recent paper by Mahanonda et al, atenolol caused substantial improvement in symptom recurrence in a randomised controlled trial of 42 patients, when given at $50-100 \mathrm{mg}$ daily. ${ }^{26}$ In this study, $62 \%$ of patients had a negative HUT on atenolol compared to $5 \%$ on placebo; this is a much higher efficacy than the $38 \%$ reported by Fitzpatrick et al. ${ }^{8}$ The likelihood of dose related differences in HUT response on atenolol was not investigated by Mahanonda et al; nonetheless it may be a reasonable explanation for these differences. ${ }^{26}$

It is interesting to note that drugs such as metoprolol and clonidine may cause a change in the pattern of presentation of the vasovagal reflex, even when they are not effective at abolishing it. In fact three out of 14 patients with vasodepressive HUT at baseline had a mixed HUT on clonidine, in our experience. This could reflect spontaneous variability of the autonomic reflex, but it seems more likely to be due to the drug effect on the sinus node, causing additional bradycardia if vagal hyperactivity occurs. This has also been shown for metoprolol in a case report by Dangovian et al, who observed conversion from a mixed response at baseline HUT to prolonged asystole during metoprolol treatment at 100 $\mathrm{mg} /$ day. ${ }^{27}$ Such an adverse effect has never been observed in our patients, irrespective of the response at baseline HUT. This observation seems to underline the potentially harmful effect of antiadrenergic drugs when underdosed: if prevention of the vasovagal reflex does not occur, the pharmacological effect may cause the vagal hyperactivity to be much more cardioinhibitory. This implies that, in order to reach the highest therapeutic index these drugs should be prescribed at highest tolerated dose, since this offers the optimal efficacy and the same risks as at lower doses.

\section{CHRONIC PHASE}

During long term follow up, no syncopal episodes occurred during a consistent evaluation time of 15 (3) (range 11 to 24) months. One might speculate that this reflects the improved capability of patients to abort syncope, although most of our patients with traumatic syncope had no prodromal symptoms, and sudden loss of consciousness occurred either during clinical episodes or at baseline HUT. However, the striking observation that rules out any improved capability to abort syncope and a possible "placebo" effect is the dramatic reduction in the number of near-syn- 
copes. This was clinically highly significant, and reflects prevention of triggering of the vasovagal reflex. This finding is clinically significant, since it confirms the value of "HUT guided treatment" as a reliable means of treating patients with vasovagal syncope, due to the high reproducibility of tilt up testing. ${ }^{15-17}$ In fact, the absence of symptom recurrence after conversion to a negative HUT during pharmacological treatment has been reported previously by Grubb et al. ${ }^{20}$ Whether this is due to the peripheral or to the central blocking effects of metoprolol cannot be determined from our data.

Patient compliance during follow up was very good, which was probably related to the great improvement in quality of life. Early side effects during the acute phase were limited by progressive dose titration, and only two patients complained of mild fatigue on some occasions during long term treatment.

\section{CONCLUSIONS}

Treatment guided by head up tilting is a reliable method of treating severely symptomatic and high risk patients with malignant vasovagal syndrome. Metoprolol is a very effective long term treatment for preventing recurrence of syncope, and high doses are recommended for optimal efficacy; a careful dose titration period helps to minimise drop out and side effects. Although there is strong pathophysiological support for its use, clonidine seems clinically less effective than metoprolol. However, clonidine may be considered a useful alternative treatment when $\beta$ blockade is contraindicated, since beneficial effects are observed in terms of time to syncope and severity of hypotension. Further studies are necessary to investigate the underlying mechanism of action of metoprolol in patients with malignant vasovagal syncope.

1 Weissler AM, Warren JV. Syncope: pathophysiology and differential diagnosis. In: Hurst JW, Logue RB, Rackley $\mathrm{CE}$, et al, eds. The heart. New York: McGraw Hill, 1986:507-29.

2 Kapoor WN. Diagnostic evaluation of syncope. Am $\mathcal{f}$ Med 1991;90:91-106.

3 Sutton R. Vasovagal syncope: clinical presentation, classification and management. In: Aubert $\mathrm{AE}$, Ector $\mathrm{H}$, Stroobandt $\mathrm{R}$, eds. Cardiac pacing and electrophysiology. $A$ bridge to the 21st century. Dordrecht: Kluwer, 1994:15-22.

4 Sra JS, Jazayeri MR, Avitall B, Akhtar M. Comparison of cardiac pacing with drug therapy in the treatment of neu-
rocardiogenic (vasovagal) syncope with bradycardia or asystole. N Engl f Med 1993;328:1085-90.

5 Sra JS, Murthy VS, Jazayeri MR, Shen Y-H, Troup PJ, Avitall $\mathrm{B}$, et al. Use of intravenous esmolol to predict efficacy of oral beta-adrenergic blocker therapy in patients with neurocardiogenic syncope. $₹$ Am Coll Cardiol 1992; 19:402-8.

6 Petersen MEV, Chamberlain-Webber R, Fitzpatrick AP, Ingram A, Williams $T$, Sutton R. Permanent pacing for cardioinhibitory malignant vasovagal syndrome. $\mathrm{Br}$ Heart f 1994;71:274-81.

7 Milstein S, Beutikofer J, Dunnigan A, Benditt DG, Gornick C, Reyes WJ. Usefulness of disopyramide for prevention of upright tilt-induced hypotension-bradycarpra. Am ₹ Cardiol 1990;65:1339-44.

8 Fitzpatrick AP, Ahmed R, Williams S, Sutton R. A randomized trial of medical therapy in "Malignant Vasovagal Syndrome" or "Neurally-mediated Bradycardia/ vagal Syndrome" or "Neurally-mediated Bradycardia/ Electrophysiol 1991;2:99-102.

9 Abi-Samra F, Maloney JD, Fouad-Tarazi FM, Astle LW. The usefulness of head-up tilt testing and hemodynamic investigation in the workup of syncope of unknown origin. PACE 1988;11:1202-14

10 Sra JS, Anderson AJ, Sheikh SH, Avitall B, Tehan PS, जै Tromp PS, et al. Unexplained syncope evaluated by electrophysiologic studies and head-up tilt testing. Ann Intern Med 1991;114:1013-9.

11 Grubb BP, Gerard G, Roush K, Temesy-Armus P, Elliot $\mathrm{L}$, Hahn $\mathrm{H}$, et al. Differentiation of convulsive syncope and epilepsy with head-up tilt testing. Ann Intern Med 1991;115:871-6.

12 Grubb BP, Temesy-Armus P, Hahn H, Elliot L. Utility of up-right tilt table testing in the evaluation and management of syncope of unknown origin. Am $\mathcal{F}$ Med 1991; ment of

13 Robertson D, Goldberg MR, Hollister AS, Wade D, Robertson RM. Clonidine raises blood pressure in severe idiopathic orthostatic hypotension. Am f Med 1983;74: 193-9

14 Sutton $R$, Petersen MEV, Brignole $M$, Raviele A, Menozzi C, Giarri P. Proposed classification for tilt-induced vasovagal syncope. Eur f Cardiac Pacing Electrophysiol 1992;2: 109-13.

15 Chen XC, Chen MY, Remole S, Kobayashi Y, Dunnigan $\infty$ A, Milstein S, et al. Reproducibility of head-up tilt table testing for eliciting susceptibility to neurally mediated syncope in patients without structural heart disease. $A m \mathcal{F}$ Cardiol 1992;69:755-60.

16 Sheldon R, Spalwinski J, Killman S. Reproducibility of isoproterenol tilt table test results in patients with syncope. Am $\mathcal{f}$ Cardiol 1992;69:1300-5.

17 Grubb BP, Wolfe D, Temesy-Armos P, Hahn H, Elliot L. Reproducibility of head-upright tilt table results in $\vec{C}$ patients with syncope. PACE 1992;15:1477-81.

18 Natale A, Akhtar M, Jazayeri M, Jazayeri M, Dhala A, Blanck Z, et al. Provocation of hypotension during headBlanck $Z$, et al. Provocation of hypotension during head-
up tilt testing in subjects with no history of syncope or presyncope. Circulation 1995;92:54-8.

19 Sheldon R, Rose S, Flanagan P, Koshman ML, Killam S. Risk factors for syncope recurrence after a positive tilttable test in patients with syncope. Circulation 1996;93:

20 Grubb BP, Temesy-Armos P, Moore J, Wolfe D, Hahn H, Elliot L. Head-upright tilt-table testing in evaluation and $\vec{O}$ management of the malignant vasovagal syndrome. $A m \mathcal{F}$ Cardiol 1992;69:904-8.

21 Hjorth S. Penbutalol as a blocker of central 5-HY1A receptor mediated responses. Eur ff Pharmacol 1992;222: 121-7.

22 Hjorth S, Sharp T. In vivo microdialysis evidence for central serotonin $1 \mathrm{~A}$ and $1 \mathrm{~B}$ autoreceptor of the beta receptor antagonist penbutalol. $¥$ Pharmacol Exp Ther 1993;265: 707-12.

23 Baum T, Shropshire AT. Inhibition of efferent sympathetic nerve activity by 5-hydroxytryptophan in centrally administered 5-hydroxytryptamine. Neuropharmacology 1975;14:227-33.

24 Tadepelli A, Mills E, Schanberg S. Central depressor of carotid baroreceptor pressor response, arterial pressure $\mathrm{O}$ and heart rate by 5-hydroxytryptophan: influence of the supramolecular areas of the brain. F Pharmacol Exp Ther 25 Grubb BP, Kosinski DJ. Serotonin and syncope: an emerg5:306-14.

26 Mahanonda N, Bhuripanyo K, Kangkagate C, Wansanit K, N Kulchot B, Nademanee $\mathrm{K}$, et al. Randomized doubleblind, placebo-controlled trial of oral atenolol in patients with unexplained syncope and positive upright tilt table $\sigma$ test results. Am Heart F 1995;130:1250-3.

27 Dangovian HI, Jarandilla R, Frumin H. Prolonged asystole during head-up tilt table testing after beta-blockade. PACE 1992;15:14-16.

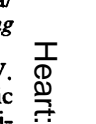

\author{
s.
}

